MATEC Web of Conferences 24,01003 (2015)

DOI: $10.1051 /$ matec conf/ 20152401003

(C) Owned by the authors, published by EDP Sciences, 2015

\title{
Structural Identification and Monitoring based on Uncertain/Limited Information
}

\author{
Eleni N. Chatzi ${ }^{1, a}$, and Minas D. Spiridonakos ${ }^{1}$ \\ ${ }^{1}$ Institute of Structural Engineering, Department of Civil, Environmental and Geomatic Engineering, ETH Zürich
}

\begin{abstract}
The goal of the present study is to propose a structural identification framework able to exploit both vibrational response and operational condition information in extracting structural models, able to represent the systemspecific structural behavior in its complete operational spectrum. In doing so, a scheme need be derived for the extraction of salient features, which are indicative of structural condition. Such a scheme should account for variations attributed to operational effects, such as environmental and operational load variations, and which likely lie within regular structural condition bounds, versus variations which indicate short- or long-term damage effects. The latter may be achieved via coupling of sparse, yet diverse, monitoring information with appropriate stochastic tools, able to infer the underlying dependences between the monitored input and output data. This in turn allows for extraction of quantities, or features, relating to structural condition, which may further be utilized as performance indicators. The computational tool developed herein for realizing such a framework, termed the PCE-ICA scheme, is based on the use of Polynomial Chaos Expansion (PCE) tool, along with an Independent Component Analysis (ICA) algorithm. The benefits of additionally fusing a data-driven system model will further be discussed for the case of complex structural response. The method is assessed via implementation on field data acquired from diverse structural systems, namely a benchmark bridge case study and a wind turbine tower structure, revealing a robust condition assessment tool.
\end{abstract}

\section{Introduction}

The realization of an ageing infrastructure demographic, underlined by certain pronounced failures, has in recent years brought forth the eminent need for efficient infrastructure management and life-cycle assessment strategies. The latter however comprises a non-trivial task, further hardened by the uncertainties entering every stage of the analysis of structural systems, including the loading (input), response (output) and even the model of the structure under investigation. In curbing the associated set of polymorphic uncertainties, it is of the essence to exploit feedback from the structure itself in the form of monitored data gathered via appropriate sensor deployments. To this end, Structural Health Monitoring (SHM) has in recent years propelled into an important area of research, dealing with the timely problem of structural condition assessment.

Amongst available SHM regimes, vibration-based methods have evolved into one of the fastest growing research areas, bearing the promise of damage detection and potential localization [1]. However, such methods are still far from being successfully implemented in largescale civil structures due to a variety of factors, including lack of precise description of loads, inadequate or overly simplified simulation models, as well as due to the susceptibility of these structures to uncontrollable influences, such as solar irradiation, temporal temperature gradients, humidity, and others [2,3]. For common structural systems, whose response may be grossly assumed as linear and stationary, Operational Modal Analysis (OMA) methods [4] offer a robust output-only identification procedure for inferring structural characteristics that are indicative of its condition, such as natural frequencies, modal shapes, and damping. However, even for the case of conventional systems, the tackling of influences induced by externally operational agents is still under research and in focus of a number of recent studies.

In related work, Alampalli [6] identifies natural frequency shifts of up to $50 \%$ for an abandoned bridge in Claverack (NY) justifying this notable variation as a consequence of the freezing of the bridge supports. While the former study addresses long-term variations, Farrar et al. [5] report a variation of the eigen-frequencies of the Alamosa Canyon Bridge of approximately $5 \%$ over the course of a single day owing to varying spatial temperature gradients. Deviations of the order of $14-18 \%$ have also been reported for the first four natural frequencies of the Z24 bridge, a benchmark study in Switzerland [4], also addressed herein . The effects of ambient temperature are further stressed in the study of Catbas et al. [7] within the context of structural reliability of long-span truss bridges, while Cross et al. [8] introduce further influencing agents into the analysis,

\footnotetext{
${ }^{a}$ Corresponding author: chatzi@ibk.baug.ethz.ch
} 
including traffic loading and wind speed, while monitoring the modal properties of the Tamar (UK) suspension bridge. Finally, beyond transportation infrastructure, Yuen and Kuok discuss the notable relationship between natural frequencies and environmental factors, namely temperature and humidity, within the context of tall building infrastructure [9].

In inferring the underlying links between structural response and the aforementioned influences, and further extracting features tied to irregular (or damaged) states, available approaches may be primarily classified in two categories:

i) methods attempting to remove the environmental/operational load signatures from vibration measurements or associated condition indices (e.g. modal properties) [10], and

ii) methods inferring a functional dependence between the measured vibration data and/or the extracted structural properties with respect to the evolution of the influencing agents [4,9]. This study follows the second approach, since the latter is capable of providing additional insight into the mechanism of the variation of structural properties under operational conditions, even for potentially unobserved sources of influence.

The proposed methodological framework is organized in three major steps:

iii) In a first identification (Id) stage, suitable data driven identification methods are employed for extracting characteristic properties of the healthy, or regularly operating, system. This is a relatively straightforward task when dealing with stationary systems (commonly bridges and buildings will be regarded as such), tackled by widely implemented OMA techniques. When however dealing with systems that are nonstationary in nature, such as Wind Turbine facilities, the task of extracting response characteristics is a nontrivial one. In this case, it is important to enforce an identification procedure that is capable of capturing the underlying physics of the system at hand. To this end, it is herein demonstrated how time varying methods may be implemented, for tracking the characteristics of complex systems [24].

iv) In a second training stage, the extracted characteristic quantities (outputs) are projected onto the space of the externally acting agents (inputs) via a polynomial chaos expansion (PCE) [19] tool. In this way, the dependences on measured environmental and operational conditions are formalized via a functional representation, which can be regarded as a metamodel able to account for the probabilistic traits of the measured input and output data. As an added step, a feature extraction algorithm, such as an Independent Component Analysis (ICA) tool [21] may be employed for producing reduced dimensionality condition indicators. v) In a final tracking stage, the evolution of the PCE-ICA estimated indicators is employed for condition assessment and potential damage/deterioration detection.

In order to illustrate the workings of the method, the proposed framework is applied on a series of data from actual structural systems, including the benchmark SHM problem of the Z24-bridge in Switzerland [4,20], and a monitored Wind Turbine tower structure in Lübbenau Germany. The results of these studies demonstrate a tool, which may reliably be adopted for condition monitoring of large-scale real world structures operating in a wide range of environments.

\section{The PCE-ICA Identification framework}

The goal of the proposed approach lies in achieving a global representation of a structural system, providing an accurate description of structural dynamics for a range of operational conditions. The proposed approach is therefore a long-term one, relying on a set of training data, which should account for a broad range of the variation of the influential (input) quantity.

\subsection{Id Stage: Inferring system characteristics}

In a first step, so-called short-term monitoring methodologies need be employed for instantaneous extraction of structural properties, i.e., of the influenced (output) quantities. In what is presented herein this is carried out in two main regimes, according to the nature of the response of the monitored structure:

\section{a) Systems described by Stationary Response}

This is a topic widely treated in the literature, with a substantial toolkit of operational modal analysis methods already available. OMA techniques extract modal characteristics relying exclusively on measurements of operational response. In such a scheme, the unmeasured ambient forces are commonly assumed as white noise sequences.

Two major classes of OMA methods may be identified, namely those formulated in the time domain and those formulated in the frequency domain. Time domain methods are commonly implemented in combination with the Natural Excitation Technique (NExT) [11]. NExT relies on the use of correlation functions of the random response of the structure when subjected to ambient (natural) excitation. The idea behind such an implementation lies in the realization that the responses of the system are uncorrelated to the disturbance, resulting a zero cross-correlation. A popular implementation of this class is the Eigensystem Realization Algorithm (ERA) coupled with NExT. Alternatively, Auto-Regressive and Stochastic Subspace type procedures may also be employed. On the other hand, frequency domain OMA methods essentially rely on the relationship between the 
input/output power spectrum density (PSD) for the case of a stochastic process.

Amongst a multiplicity of available works overviewing this topic, Zhang and Brinchker [12], Reynders and De Roeck [20] and Peeters and De Roeck [14] provide an extensive review on established techniques, while Magalhaes and Cunha [15] provide an informative tutorial to OMA based on bridge vibration data.

\section{b) Systems described by Non-Stationary Response}

A number of systems, including wind turbine structures, which are herein explored in the applications section, exhibit non-stationary short-term dynamics. Within this context, Wavelet-based [29] or Time-varying AutoRegressive Moving Average (TARMA) models constitute suitable tools for tracking the time evolution of the involved dynamics by means of a compact parametric formulation.

In the case of TARMA models, we may define such a model of AR/MA order equal to $n$ via the following equation:

$y[t]+a_{1}[t] y[t-1]+\ldots+a_{n}[t] y[t-n]=$

$=e[t]+c_{1}[t] \mathrm{e}[t-1]+\ldots+c_{n}[t] e[t-n], e[t] \sim \mathrm{NID}\left(0, \sigma_{e}^{2}[t]\right)$

where $t$ designates discrete time, $y[t]$ denotes the observed non-stationary signal, $e[t]$ the residual sequence, corresponding to the unmodeled part of the signal, which is assumed to be normally identically distributed with zero mean and time-varying variance $\sigma_{e}[t]$ while $a_{i}[t], c_{i}[t]$ are the time-varying AR and MA parameters, respectively.

Since the aim is to utilize models which are time varying in nature, Smoothness Priors models may be employed, comprising stochastic difference equations, which govern the evolution of each of the AR and MA parameters [17]:

$$
\begin{array}{ll}
(1-B)^{\kappa} a_{i}[t]=w_{a_{i}}[t], & w_{a_{i}}[t] \sim \operatorname{NID}\left(0, \sigma_{w_{a}}^{2}[t]\right) \\
(1-B)^{\kappa} c_{i}[t]=w_{c_{i}}[t], & w_{c_{i}}[t] \sim \operatorname{NID}\left(0, \sigma_{w_{c}}^{2}[t]\right)
\end{array}
$$

where $B$ is the backshift operator $\left(B^{\kappa} x[t]=x[t-\kappa]\right), \kappa$ designates the difference equation order, and $w_{i}[t]$ zeromean, uncorrelated, mutually uncorrelated and also uncrosscorrelated with $e[t]$, Gaussian sequences with time-dependent variance.

The smoothness of evolution of the AR/MA parameters is controlled by the order of the difference equation constraints $\kappa$, and the variance $\sigma_{w_{i}}^{2}[t]-$ where a small variance indicates smooth evolution of the corresponding time-varying parameter and vice-versa.

In order to illustrate the model parameter estimation through a simple example, consider the $\operatorname{TAR}(n)$ case and a second order $(\kappa=2)$ stochastic smoothness constraint:

$$
\begin{aligned}
& (1-B)^{2} a_{i}[t]=w_{a_{i}}[t] \Rightarrow \\
& a_{i}[t]=2 \cdot a_{i}[t-1]-a_{i}[t-2]+w_{a_{i}}[t]
\end{aligned}
$$

The constraints corresponding to all AR parameters may be formulated as:

$$
\begin{aligned}
{\left[\begin{array}{c}
a_{1}[t] \\
\vdots \\
a_{n}[t] \\
a_{1}[t-1] \\
\vdots \\
a_{n}[t-1]
\end{array}\right] } & {\left[\begin{array}{cccccc}
2 & \ldots & 0 & -1 & \ldots & 0 \\
\vdots & \ddots & \vdots & \vdots & \ddots & \vdots \\
0 & \ldots & 2 & 0 & \ldots & -1 \\
1 & \ldots & 0 & 0 & \ldots & 0 \\
\vdots & \ddots & \vdots & \vdots & \ddots & \vdots \\
0 & \ldots & 1 & 0 & \ldots & 0
\end{array}\right] \cdot\left[\begin{array}{c}
a_{1}[t-1] \\
\vdots \\
a_{n}[t-1] \\
a_{1}[t-2] \\
\vdots \\
a_{n}[t-2]
\end{array}\right]+} \\
& {\left[\begin{array}{ccc}
1 & \ldots & 0 \\
\vdots & \ddots & \vdots \\
0 & \ldots & 1 \\
0 & \ldots & 0 \\
\vdots & \ddots & \vdots \\
0 & \ldots & 0
\end{array}\right] \cdot\left[\begin{array}{c}
w_{a_{1}}[t] \\
\vdots \\
w_{a_{n}}[t]
\end{array}\right] }
\end{aligned}
$$

In a similar manner, it may be demonstrated that the general $(\kappa$-th order) smoothness constraint of Equation (2) can be expressed as:

$$
\mathbf{z}[t]=\mathbf{F} \cdot \mathbf{z}[t-1]+\mathbf{G} \cdot \mathbf{w}[t]
$$

with

$$
\begin{aligned}
& \mathbf{z}[t] \triangleq\left[a_{1}[t] \ldots a_{n}[t] \vdots \ldots \vdots a_{1}[t-\kappa+1] \ldots a_{n}[t-\kappa+1]\right]_{\kappa \cdot n \times 1}^{T} \\
& \mathbf{w}[t] \triangleq\left[w_{a_{1}}[t] w_{a_{2}}[t] \ldots w_{a_{n}}[t]\right]_{n \times 1}^{T}
\end{aligned}
$$

and $\mathbf{F}, \mathbf{G}$ are matrices of the following forms (depending on the value of the order $\kappa$ ):

$$
\begin{aligned}
& \boldsymbol{\kappa}=1: \\
& \mathbf{F} \triangleq \mathbf{I}_{n}, \quad \mathbf{G} \triangleq \mathbf{I}_{n}
\end{aligned}
$$

$$
\begin{aligned}
& \boldsymbol{\kappa}=2: \\
& \mathbf{F} \triangleq\left[\begin{array}{cc}
2 \cdot \mathbf{I}_{n} & -\mathbf{I}_{n} \\
\mathbf{I}_{n} & \mathbf{0}_{n}
\end{array}\right], \quad \mathbf{G} \triangleq\left[\begin{array}{l}
\mathbf{I}_{n} \\
\mathbf{0}_{n}
\end{array}\right]
\end{aligned}
$$

$$
\begin{aligned}
& \boldsymbol{\kappa}=3: \\
& \mathbf{F} \triangleq\left[\begin{array}{ccc}
3 \cdot \mathbf{I}_{n} & -3 \cdot \mathbf{I}_{n} & \mathbf{I}_{n} \\
\mathbf{I}_{n} & \mathbf{0}_{n} & \mathbf{0}_{n} \\
\mathbf{0}_{n} & \mathbf{I}_{n} & \mathbf{0}_{n}
\end{array}\right], \quad \mathbf{G} \triangleq\left[\begin{array}{l}
\mathbf{I}_{n} \\
\mathbf{0}_{n} \\
\mathbf{0}_{n}
\end{array}\right]
\end{aligned}
$$

and so on,

where $\mathbf{I}_{n}, \mathbf{0}_{n}$ designate the $n \times n$ dimensional identity and zero matrices, respectively. As indicated by the above 
expressions, $\mathbf{z}[t]$ forms a state vector, whereas $\mathbf{w}[t]$ consists of the residuals entering in each constraint expression. The TAR(n) representation may then be expressed as:

$$
x[t]=\mathbf{h}[t]^{T} \cdot \mathbf{z}[t]+e[t]
$$

with:

$$
\mathbf{h}[t]=[-x[t-1] \ldots-x[t-n] \vdots 0 \ldots 0]]_{\kappa \cdot n \times 1}^{T}
$$

Thus, the SP-TAR(n) model may be completely expressed in the state space form summarized via Equations (5), (8). Its estimation may consequently be achieved via the Kalman Filter (KF) approach for given $\kappa$ order and residual variance ratio $v=\sigma_{w}^{2}[\mathrm{t}] / \sigma_{e}^{2}[\mathrm{t}]$, which is considered to be constant for all time instants $t$. However, the full SP-TARMA case results in a nonlinear state estimation problem due to the unknown residual sequence of $e[t]$ in $\mathbf{h}[t]$. For this reason, the Extended Least Squares (ELS) algorithm is presently employed for the recursive estimation of the state vector $\mathbf{z}[t]$. Since further details on the method are beyond the scope of this paper, the interested reader is referred to the work of Spiridonakos et al. [16] for a deeper insight into the above formulation.

The model inferred through this process will be referred to as "short-term" in the sense that it may represent the WT dynamics for a given set of observed conditions. The ability to predict the response of the system through such a parametric, and therefore low-cost, simulation model lays the ground for the subsequent derivation of metrics for detecting abnormalities/damage.

\subsection{Training Stage: Inferring input-output relationship}

The previously described short-term monitoring methods enable, in a first step, extraction of the response characteristics of the healthy structure. Once these are attained, a polynomial chaos expansion (PCE) method [18], [19] is further applied for the projection of these output response estimates on the probability space of the measured operational conditions. A short overview of the employed mathematical framework is provided in this section. For further details, the interested reader is pointed to previous works of the authoring team [25]-[29].

\subsubsection{Polynomial Chaos Expansion (PCE)}

PCE concerns the expansion of a random output variable on polynomial chaos basis functions, which are orthonormal to the probability space of the system's random inputs. Consider a system $\mathcal{S}$ comprising $\mathrm{M}$ random input parameters adequately represented by a set of independent random variables $\left\{\Xi_{1}, \Xi_{2}, \ldots, \Xi_{M}\right\}$. The latter set may for example pertain to temperatures measured at different locations of the structure, gathered in a random vector $\boldsymbol{\Xi}$ of prescribed joint Probability Density Function (PDF) $p_{\Xi}(\xi)$ [19]. The resulting structural output denoted by $\mathbf{Y}=\mathcal{S}(\boldsymbol{\Xi})$ corresponding for instance to modal frequencies, will also be random. Provided that $\mathbf{Y}$ has finite variance, it can be expressed in the following form:

$$
\mathbf{Y}=\mathcal{S}(\boldsymbol{\Xi})=\sum_{\mathbf{d} \in \mathbb{N}^{M}} \boldsymbol{\theta}_{\mathbf{d}} \boldsymbol{\varphi}_{\mathbf{d}}(\boldsymbol{\Xi})
$$

where $\boldsymbol{\theta}_{\mathbf{d}}$ are unknown deterministic coefficients of projection, $\mathbf{d}$ is the vector of multi-indices of the multivariate polynomial basis, and $\boldsymbol{\varphi}_{\mathbf{d}}(\boldsymbol{\Xi})$ are the polynomial basis (PC) functions orthonormal to $p_{\Xi}(\xi)$. These basis functions result as tensor products of the corresponding univariate functions [19]. Each univariate probability density function may be associated with a wellknown family of orthogonal polynomials. For instance, the normal distribution is associated with Hermite polynomials while the uniform distribution with Legendre (Table 1). A list of the most common probability density functions along with the corresponding orthogonal polynomials and the relations for their construction may be found in [22].

Table 1.Types of Wiener-Askey polynomial chaos and their underlying random variables.

\begin{tabular}{|c|c|c|}
\hline PDF & Support & Polynomials \\
\hline $\begin{array}{c}\text { Normal } \\
(\text { Gaussian })\end{array}$ & $(-\infty, \infty)$ & Hermite \\
\hline Gamma & {$[0, \infty)$} & Laguerre \\
\hline Beta & {$[0,1]$} & Jacobi \\
\hline Uniform & {$[-1,1]$} & Legendre \\
\hline
\end{tabular}

For implementation purposes, the basis functions series need be truncated to a finite number of terms, with the usual approach consisted in selection of the multivariate polynomial basis with total maximum degree $\left|d_{j}\right|=\sum_{m=1}^{M} d_{j, m} \leq p, \forall j$. When truncating the infinite series of expansion of Equation (10) to the first $p$ terms, the resulting PCE model is fully parametrized in terms of a finite number of deterministic coefficients of projection $\boldsymbol{\theta}_{\mathbf{d}}$. The parameter vector $\boldsymbol{\theta}_{\mathbf{d}}$ may be estimated by solving Equation (10) in a least squares sense. Toward this end, the data collected on the sytems's output response and the probabilistic information (PDFs) available on the input variables are employed. The PDFs of the input variables may be obtained by fitting known statistical distributions to the observed input variable datasets.

Considering for example a structural system subjected to ambient excitation which is susceptible to changes of the environmental conditions, a given output variable, could consist in estimates of its first natural frequency (as derived from the data). The evolution of this output variable could be expanded on a PC basis constructed to be orthogonal to the experimentally estimated PDF of 
temperature data. In this way, an indication is obtained on the sensitivity of the natural frequencies of the structure to the probabilistic properties of temperature variations affecting the structural system. An implementation of this type is demonstrated in the first application provided herein, pertaining to the case of a bridge system.

\subsubsection{Independent Component Analysis (ICA)}

As aforementioned PCE relies on the assumption of independent input variables. However, when discussing environmental conditions (temperature, humidity, wind direction and velocity, and others), the common case is that a large bulk of data is in fact available, which is in some degree correlated. In order to select only a small number of independent features, which is nonetheless able to describe a large portion of this data, an Independent Component Analysis (ICA) algorithm is utilized herein.

ICA is a source separation method, which aims at estimating independent unobservable (latent) variables that are intermixed with observed quantities [21]. This is herein required for sorting out independent input variables among the possibly numerous measurements of temperature and humidity that are potentially available via a dense grid of corresponding environmental sensors.

The method relies on the assumption of $n$ observations of random variables $y_{j}(t), j=1, \ldots, n$, which are linear mixtures of $n$ independent components $s_{j}(t), j=1, \ldots, n$ as (in matrix notation):

$$
\mathbf{y}=\mathbf{A s}
$$

ICA attempts to identify the latent independent variable vector $\mathbf{s}(t)$, via a search for non-Gaussian components, in contrast to principal component analysis and other second order methods, which are based on the covariance matrix of random variables.

Within this context, ICA aims at identifying the mixing matrix A along with the vector $\mathbf{s}$ of unobserved independent variables of Equation (11), through the available information on the observed vector $\mathbf{y}$. More accurately, when inverting the mixing matrix $\mathbf{A}$, as $\mathbf{W}=\mathbf{A}^{-1}$, the vector of the independent latent variables $\mathbf{s}$ may be readily obtained as:

$$
\mathbf{s}=\mathbf{W y}
$$

As already noted, the key in estimating the ICA model is the non-Gaussianity of the unobserved sources. Therefore, ICA estimation algorithms implement nonlinear optimization methods, aiming at the maximization of the non-Gaussianity of each one of the latent variables $\mathbf{w}^{T} \mathbf{y}$, with $\mathbf{W}$ designating a column vector of matrix $\mathbf{W}$.

The adopted measure of non-Gaussianity may be based on kurtosis, negentropy, and others [21]. The details of the method may be found in [27], while a flowchart of a typical ICA algorithm implementation is outlined in Table 2.

Table 2. Flowchart of the ICA algorithm.

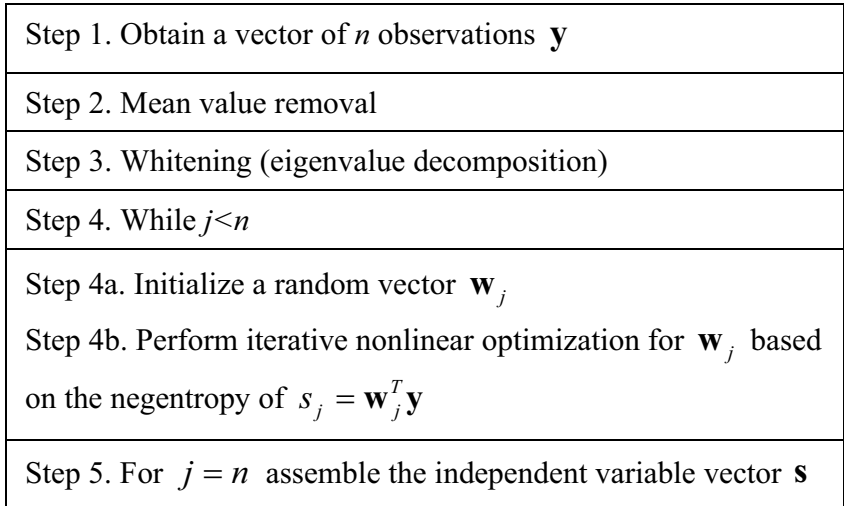

Apart from its usefulness in rendering independent input variables for feeding the PCE tool, the ICA holds one further valuable trait within the context of the proposed SHM framework, as it helps extract salient features of reduced dimensionality. Indeed, significant discussion is nowadays allocated on the storage potential of the Big Data stream resulting from long-term SHM implementations. ICA assists in reducing the data to be stored form the SHM system by enabling extraction of latent variables, i.e., of only a small number of valuable input and output features to be employed within the PCE framework.

\subsection{Tracking Stage}

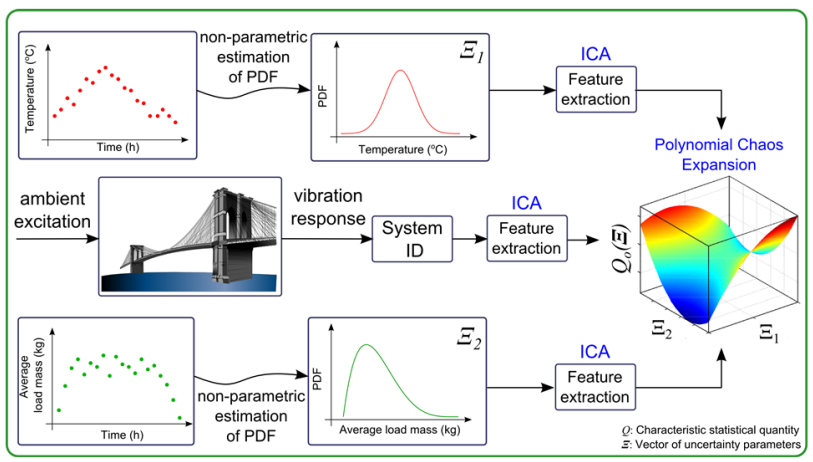

Figure 1. Schematic diagram of the PCE-ICA identification approach.

A schematic diagram of the training phase of the proposed framework is illustrated in Figure 1. Following the training phase, the PCE-ICA estimated statistical properties of the modal characteristics may be utilized in a final tracking stage for monitoring the system's condition and devising suitable damage indicators. This is performed via tracking of the error between the PCE-ICA predicted values and the actually measured ones on site. For a healthy structure within conditions of regular operation, this error is expected to be normally distributed within prescribed thresholds. If the error distribution is indeed close to normal $\sim \mathcal{N}(0, \sigma)$, an interval of $\pm 3 \sigma$ would correspond to approximately a $99.7 \%$ confidence interval around the estimated mean value. 


\section{Application Case Studies}

The workings of the method will be now demonstrated via implementation on a set of actual large-scale structures under both operating and damaged conditions.

\subsection{Implementation on a Stationary System - Z24 bridge}

In already published work [27], the proposed SHM framework has been validated using test data from another Swiss bridge, i.e., the Z24 benchmark case [20]. The Z24 bridge was monitored for a period of 9 months and finally "artificially damaged" for the purposes of the BRITEEURAM SIMCES project. The bridge was over-passing the A1 Zurich-Bern highway connecting Utzemstorf and Koppigen and its demolition was planned after the monitoring period since a new railway, adjacent to the highway, required a new bridge with a larger side span. The bridge was monitored from November 1977 till August 1998 through a permanent system acquiring hourly ambient vibration response and environmental condition measurements. More specifically, 16 acceleration sensors were installed measuring ambient vibration response. 13 of them are shown in Figure 2a, while three more were installed on one of the piers. In addition, 49 environmental condition sensors were installed for measuring air temperature, wind characteristics, humidity, bridge expansion, soil temperatures at the boundaries and bridge concrete temperatures. Data from these sensors was acquired on an hourly basis.
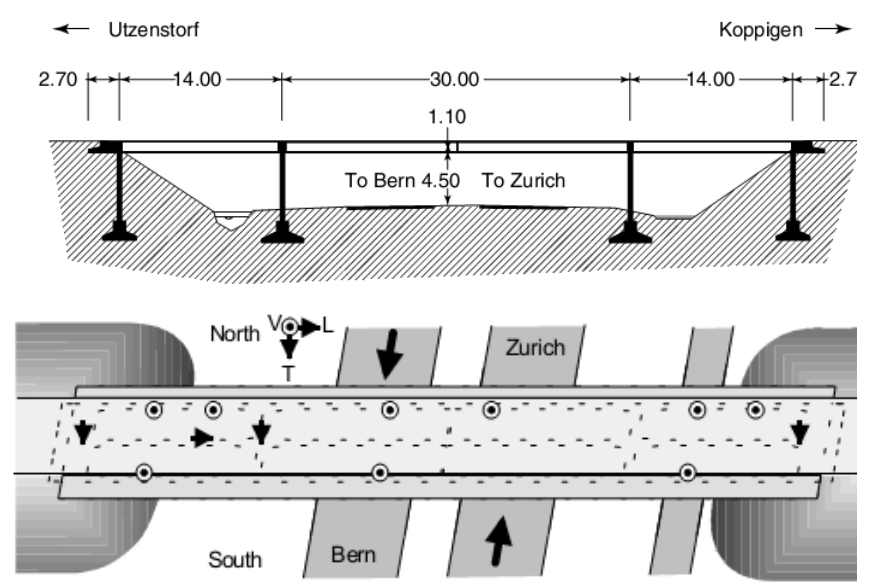

Figure 2. Z24-Bridge: longitudinal section and top view [23].
Table 3. Types of artificial damage induced on bridge Z24 [20].

\begin{tabular}{|l|l|}
\hline No & Damage type \\
\hline 1 & Lowering of pier \\
\hline 2 & Tilt of foundation \\
\hline 3 & Spalling of concrete \\
\hline 4 & Landslide at abutment \\
\hline 5 & Failure of concrete hinge \\
\hline 6 & Failure of anchor heads \\
\hline 7 & Rupture of tendons \\
\hline
\end{tabular}

After the nine-month period, progressive damages were artificially induced within one month (August 10 September 11, 1998). The monitoring system was still in operation during the demolition phase, while during the night-time vibration tests based on hammer, dropping mass device and shakers were performed. The specific types of induced damages are summarized in Table 3, while more details on the damage scenarios and description of the simulation of the real damage cause may be found in [20].

The framework described in this study has been applied on the monitoring data-stream collected from the Z24 bridge. Specifically, the temperatures measured at six locations at the center of the middle span along with the air temperature serve as input variables, or so called influencing agents. The set of output variables comprises the first four natural frequencies of the system. These are estimated through Stochastic Subspace Identification (SSI), i.e., an OMA approach (as mentioned in Section 2.1) adopted within the context of an automated modal analysis tool.

The dependence of natural frequency estimates on the temperature measured at the center of the web is plotted in Figure 3 indicating a bilinear temperature - natural frequency dependence.

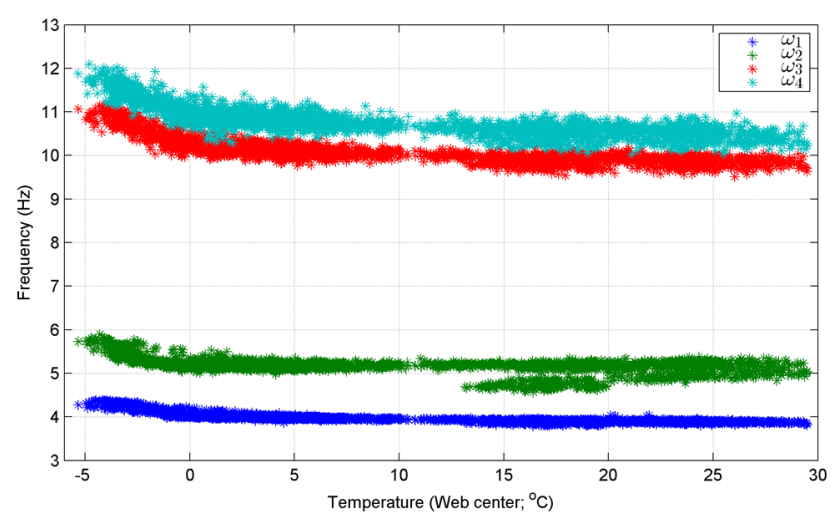

Figure 3. The first four natural frequency estimates plotted against temperature (center of the web).

Interestingly, the variation that is due to environmental conditions is in fact more pronounced than the one attributed to the damage. The influence of damage is discernable only in the plots of the second mode. A robust 
predictor tool therefore necessitates incorporation of the environmental influence effects in the simulation process.

Even though, the list of input variables affecting the structural behavior of $\mathrm{Z} 24$ is not limited to this set of temperatures, it is assumed that these are still sufficient for describing the variability of the estimated modal properties. Had the deployment permitted, the set of monitored input parameters could have been expanded to include further influences, such as wind or traffic loads.

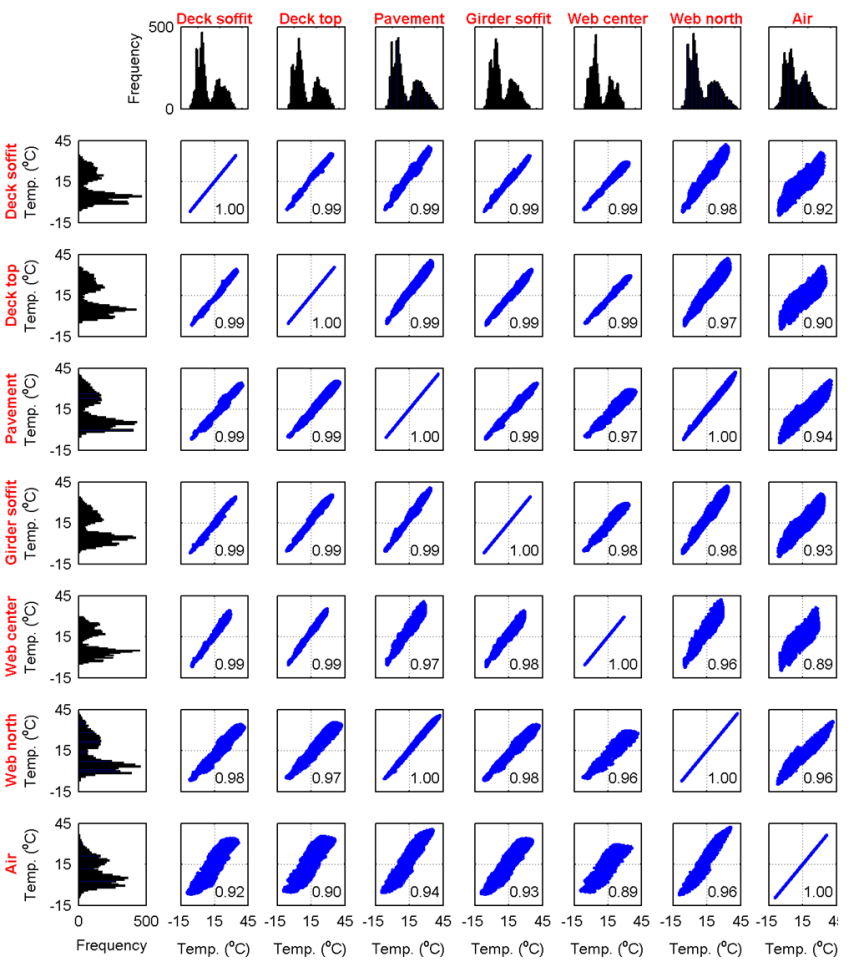

Figure 4. Histograms of the measured temperatures (upper row and left column) along with the scatter plots and the estimated correlation values.

As a first step, and before expanding the natural frequency estimates to the PC functional basis orthogonal to the PDFs of the random input variables, i.e. recorded temperatures, the independency of the latter has to be checked. Looking at the scatter plots of Figure 4, drawn for each pair of measured temperatures, it is evident that they are highly correlated (correlation larger than 0.95 for almost all pairs), while the smallest correlation is 0.89 .

Therefore, ICA has to be implemented before PCE is applied on the natural frequency estimates. The scatter plots of the corresponding ICA-based estimates of the independent random (latent) variables, are shown in Figure $\mathbf{5}$, with most of the variables indicating a correlation smaller than 0.1 , with the larger correlation being 0.13 . It should be noted that these transformed variables do not correspond to temperature anymore.

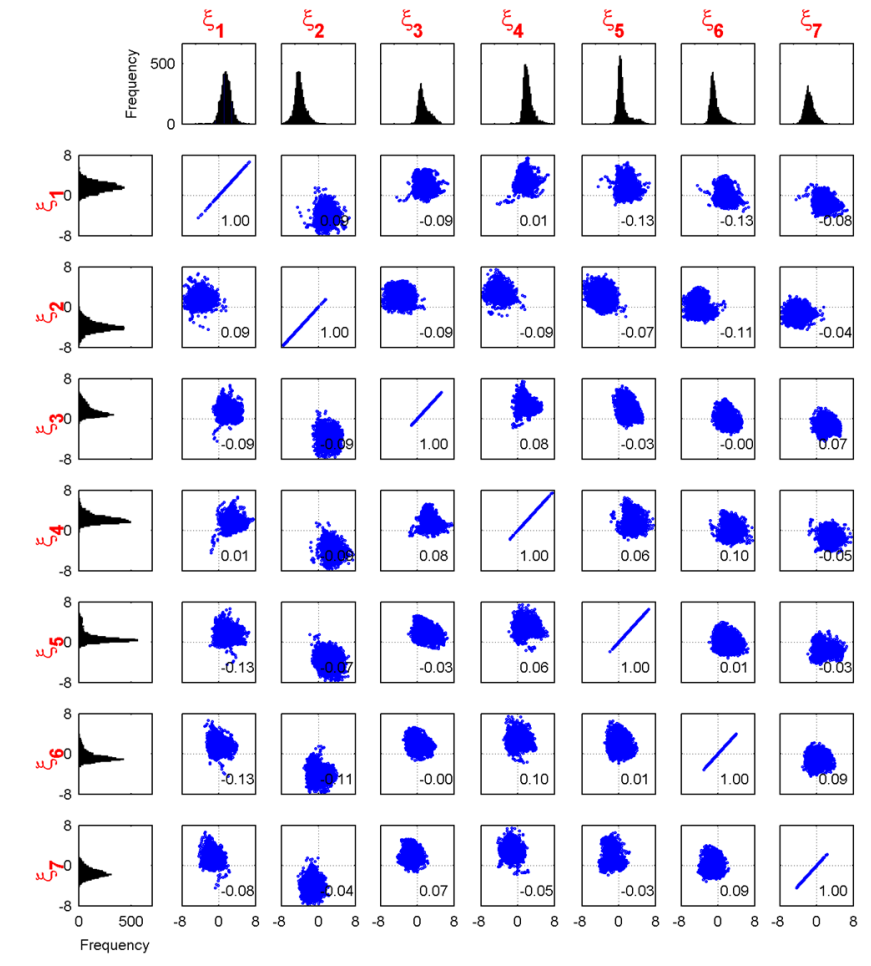

Figure 5. Histograms of the ICA-based estimated independent random variables (upper row and left column) along with the scatter plots and the estimated correlation values.

In a next step, the ICA transformed independent input variable set is fed into the PCE tool for inferring the relationship between the experimentally calculated natural frequencies and the measured temperature inputs. Toward this end, the input variables are transformed into uniformly distributed variables by using their non-parametrically estimated cumulative distribution functions and a PC basis comprising multivariate Legendre polynomials is finally constructed.

The set of natural frequency estimates is divided into an estimation and a validation set. The estimation set comprises 1500 values randomly selected from the first eight months of the monitoring period (one month before the first damage), while the rest of the values are used as validation set including the complete set recorded during the last month of the monitoring period plus the complete set of values corresponding to the period of induced damages, that is 2744 values.

It is essential that the training set includes a full seasonal cycle and therefore this framework is only meaningful within a long-term monitoring context.

Figure 6 summarizes the results of PCE-ICA approach for tracking the first four natural frequencies of the bridge. 


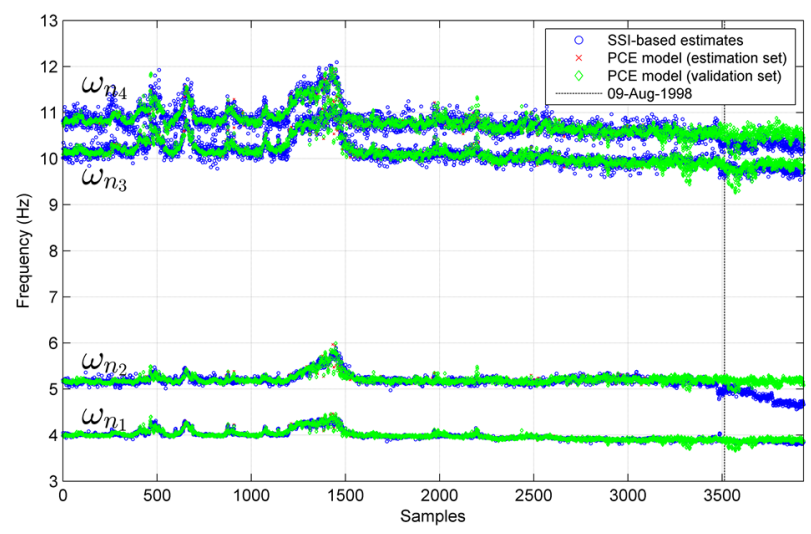

Figure 6. PCE natural frequency estimates for both the estimation and validation set contrasted to the true SSI-based estimates.

In the plot:

(a) The blue marker denotes the experimentally derived natural frequencies, via use of the SSI scheme.

(b) The red marker denotes the training set, used for deriving the PCE representation between observed inputs and outputs.

(c) The green marker designates the prediction of the PCE model. As long as the model prediction agrees with the corresponding measurements (in blue), the structural response lies within operational (regular) bounds. Deviations of the predicted from the measured quantity serve as indications of irregularity or damage.

A better way to quantify performance however is by retaining, not all, but only few, i.e., reduced dimensionality, salient features that are indicative of the response. This may once again be achieved via use of the ICA method, whereby a reduced number of independent random variables from both the vector of temperature measurements (input variables) and the estimated natural frequencies (output variables - damage index) may be inferred.

In this case, a single input latent variable is enough to describe the variance of the temperature measurements and the same applies for the vector of the natural frequencies. PCE of the output to the input (latent variables now) results in the upper plot of Figure 7, where the salient feature's PCE-ICA prediction is plotted against its "true" value as derived from actual measurements. In the lower plot of Figure 7, a single damage index may be inferred, defined as the error between measurement and prediction.

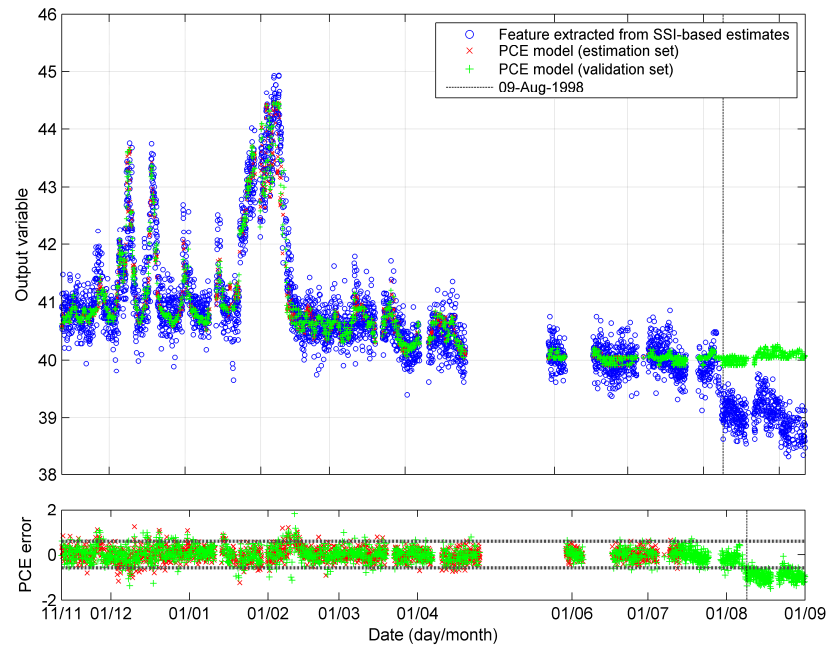

Figure 7. Extracted feature variable compared with the PCE modeling estimates (top) and the extracted damage index based on the PCE prediction errors for the Z24 benchmark case, where artificial damage is inflected after 9 months of monitoring (vertical line).

During regular operation, this error should be lie within a prescribed threshold indicated by the horizontal dashed lines in the plot. Indeed prior to the occurrence of damage (marked with a black vertical line), the error lies within the prescribed thresholds (denoted via dashed horizontal lines), apart from isolated outliers, which can be ignored as isolated points. In the period following the point where the $1^{\text {st }}$ damage is induced the error plot develops a persistent offset from the expected mean, exceeding the prescribed thresholds, which correctly translates to damage indication. In fact, the method is able to detect, also the preliminary works performed on the structure in preparation of the damage sequence, consisting in the lowering of the piers on August 7th, i.e., 3 days before the actual implementation of the designed damage scenarios (August 10th), which is marked in the Figure with a red vertical line.

The PCE-ICA monitoring tool and the extracted performance index proves therefore capable of reliably reproducing the estimation set values, while more importantly a very good accuracy is also achieved for values belonging in the validation set for the interval prior to damage.

\subsection{Implementation on a Non-Stationary System - Wind Turbine tower monitoring}

The SHM framework introduced in the previous sections is in this section applied on the case study of a real Wind Turbine (WT) under operation. The WT under study is one of the eight V90 2MW Vestas generators of a wind farm in Lübbenau in northern Germany, owned by Repower Deutschland GmbH. The vibration response of the WT were measured by triaxial accelerometers (STM LIS344ALH MEMS sensors) at five distinct locations of the WT tower (Figure 8). A 10-minute-long dataset was recorded every half hour for 29 days (from 18/18/2013 till 15/1/2014), resulting in 1392 datasets. The signals 
recorded at $200 \mathrm{~Hz}$ were low-pass filtered and downsampled to $12.5 \mathrm{~Hz}$ (cutoff frequency at $5 \mathrm{~Hz}$ ).

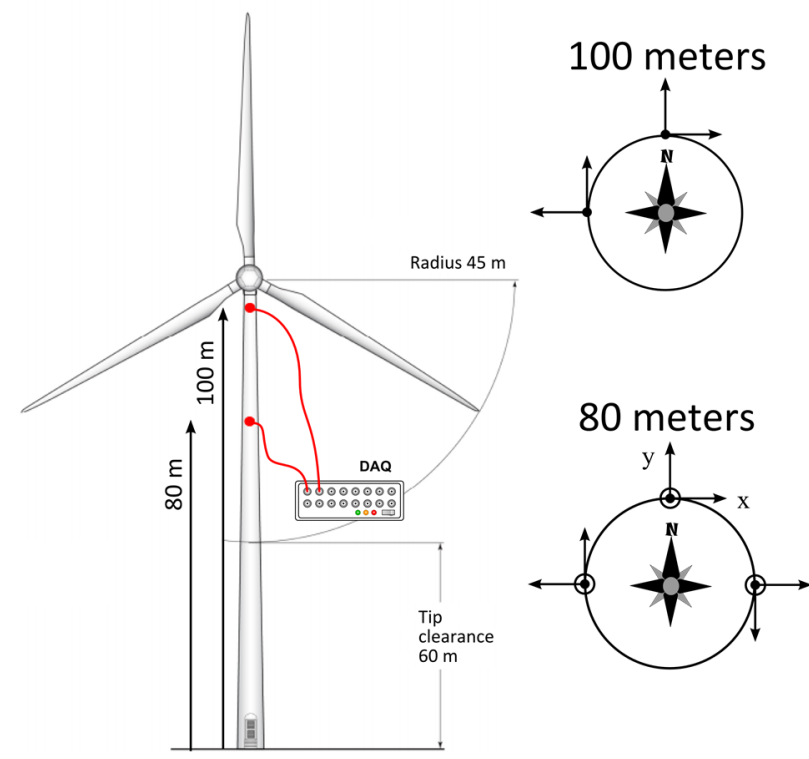

Figure 8: Schematic diagram of the experimental setup on the tower of the WT.

A dataset corresponding to the parked WT is initially used for extracting its modal properties for the purposes of comparison. Toward this end, the y-axis response measured at $80 \mathrm{~m}$ height (North sensor location; Figure 8) and a stationary ARMA method based on prediction error method are utilized. The results of a stochastic subspace identification method based on the canonical variate algorithm are also shown in Table 4 for comparison purposes.

The resulting stabilization diagram for model orders between 2 and 50 is shown in Figure 9. The final AR/MA orders are selected equal to 32 and the estimated natural frequencies and damping ratios based on ARMA model are also summarized in this figure. It should be noted, that only the natural frequencies with damping ratio less than $5 \%$ are included in the plot.

In contrast to the stationary dynamics of the parked WT the dynamics of the operating WT are susceptible to change within some minutes as clearly shown by the spectrogram of the signal recorder at 8:30 am, 26/012/2013 in Figure 10. In this figure a Short Time Fourier Transform is employed with a Hamming data window $(\mathrm{NFFT}=512$; overlap 98\%).

Furthermore, it may be clearly observed that some additional frequencies are introduced in the spectrogram due to the rotation of the WT's nacelles and blades. On the same figure, the evolution of the estimated natural frequencies based on $\operatorname{SP}-\operatorname{TARMA}(32,32)$ with smoothness constraints order $\kappa$ equal to 1 and variance ratio $v=0.01$ are shown by blue solid lines while in dashed gray lines the corresponding estimates of an conventional ARMA $(32,32)$ model are also shown. The latter are of course incapable of tracking any variability and they normally return averages of the nonstationaty natural frequencies, in contrast to the nonstationary SP-TARMA model which tracks with good accuracy the evolution of the dynamics when compared to the spectrogram shown in the background.

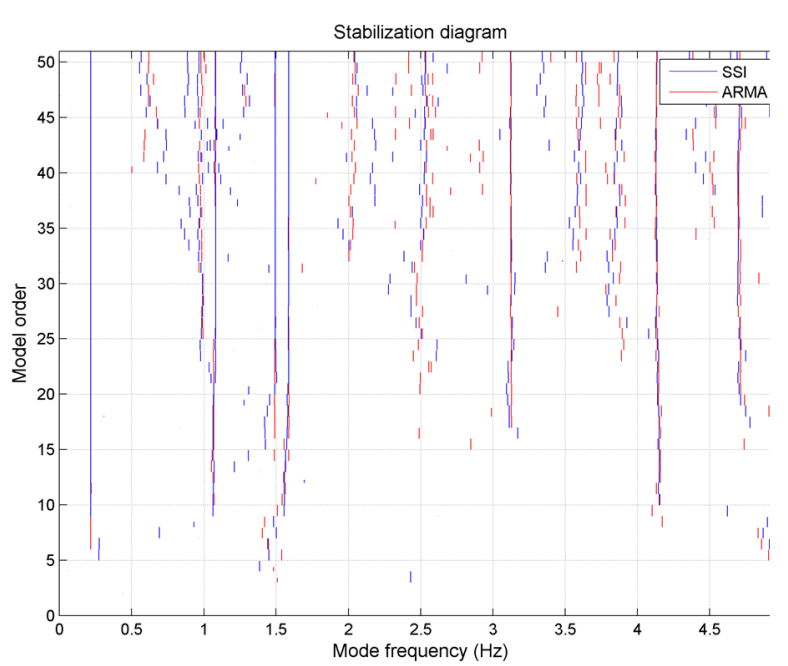

Figure 9: Dynamics of the parked WT. Stabilization plot for the stationary ARMA and SSI methods.

Table 4. Estimated natural frequencies and damping ratios based on an ARMA $(32,32)$ model.

\begin{tabular}{|c|c|c|}
\hline$\#$ & $\begin{array}{c}\text { Natural freq. } \\
\mathbf{( H z )}\end{array}$ & $\begin{array}{c}\text { Damping ratio } \\
\mathbf{( \% )}\end{array}$ \\
\hline 1 & 0.2170 & 0.2716 \\
\hline 2 & 1.0817 & 1.4520 \\
\hline 3 & 1.4927 & 0.6207 \\
\hline 4 & 1.5864 & 0.3858 \\
\hline 5 & 2.0033 & 3.3644 \\
\hline 6 & 2.5115 & 3.7262 \\
\hline 7 & 3.1251 & 0.6683 \\
\hline 8 & 3.6084 & 0.9387 \\
\hline 9 & 3.8287 & 2.4248 \\
\hline 10 & 4.1340 & 1.3802 \\
\hline 11 & 4.7153 & 1.0199 \\
\hline
\end{tabular}

The long-term monitoring framework aims at the accurate description of the dynamic properties of the structure for a range of acting, operational and environmental conditions. In this case, we refer to a variability that is different from the short-term one treated in Section 2.1b. This "longterm" variability pertains to the loading and environmental conditions which are continually changing within the range of hours, or even minutes:

i. Non-periodic, stochastic loads caused by wind gusts and turbulence (within the order of minutes)

ii. Changes in the input wind flow profile (within the order of minutes). 
iii. Environmental conditions variation (temperature, humidity, solar radiation, wave loading and others; within the order of hours).
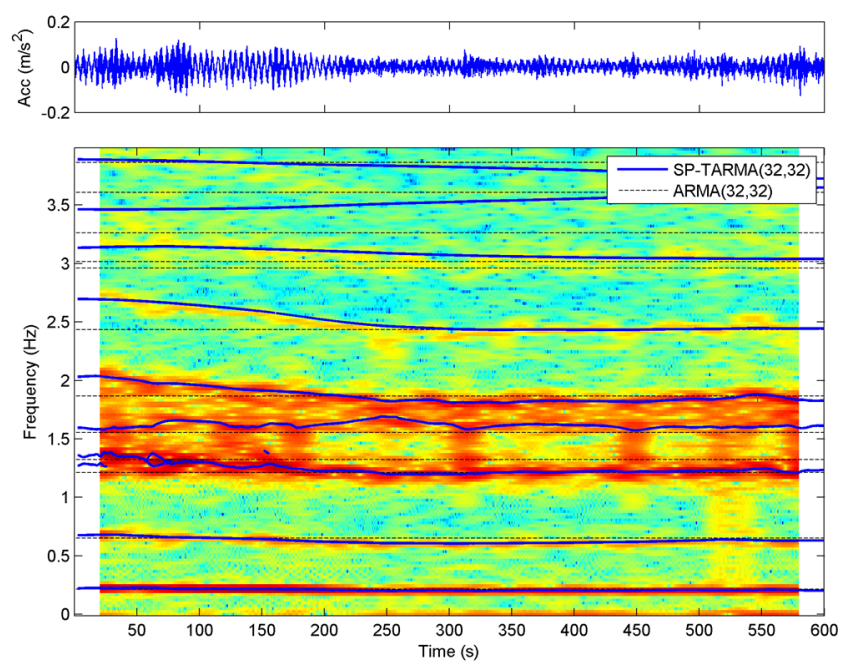

Figure 10: Dynamics of the WT under normal operation. Stationary ARMA $(32,32)$ natural frequency estimates are compared with those of the nonstationary SP-

TARMA(32,32) model (spectrogram in the background).

Within such a context, for the long-term monitoring of the system the $10 \mathrm{~min}$ average information attained from the Supervisory Control and Data Acquisition System (SCADA), installed in the nacelle, is additionally utilized. The list of available operational and environmental parameters provided via the SCADA is outlined in Table 5 with those selected for the subsequent analysis indicated in bold. An additional plot of the selected parameters is provided in Figure 11. These values correspond to the 1392 datasets of the vibration response measurements.

Since these variables are standardly available in a 10minute average format, the output of the ICA-PCE framework was also selected to be the standard deviation (std) of the SP-TARMA $(32,32)$ residuals for the 10 minute intervals, that is the mean value of the non-stationary residuals std. The input variables were transformed in uniform variables and thus the Legendre polynomials are used for the PCE while the maximum polynomial order was selected equal to seven ( 22 criterion is used for this selection).

The residuals std for each dataset and the corresponding PCE model estimates are indicated in Figure 12. It is noted that the total number of datasets is divided into a 1000-long estimation set and a 392-long validation set. The PCE errors are also shown in the lower part of Figure 12 along with the corresponding $95 \%$ confidence intervals calculated for the fitted Gaussian distribution of the estimation set errors. Evidently, a statistical hypothesis testing could be adopted in this case for damage detection, offering an automated SHM tool necessitating little or no user inference after the initial model-training period.
Table 5. List of available operational and environmental conditions through the SCADA system.

\begin{tabular}{|ll|}
\hline \multicolumn{2}{|l|}{ Variables } \\
\hline $\mathbf{1}$ & Wind direction $[\mathbf{d e g}]$ \\
\hline 2 & Min wind speed $[\mathrm{m} / \mathrm{s}]$ \\
\hline 3 & Average wind speed $[\mathrm{m} / \mathrm{s}]$ \\
\hline $\mathbf{4}$ & Max wind speed $[\mathrm{m} / \mathrm{s}]$ \\
\hline 5 & Min power $[\mathrm{kW}]$ \\
\hline $\mathbf{6}$ & Average power $[\mathbf{k W}]$ \\
\hline 7 & Max power $[\mathrm{kW}]$ \\
\hline 8 & Nacelle direction $[\mathrm{deg}]$ \\
\hline 9 & Nacelle temperature $\left[{ }^{\circ} \mathrm{C}\right]$ \\
\hline $\mathbf{1 0}$ & Ambient temperature $\left[{ }^{\circ} \mathbf{C}\right]$ \\
\hline
\end{tabular}
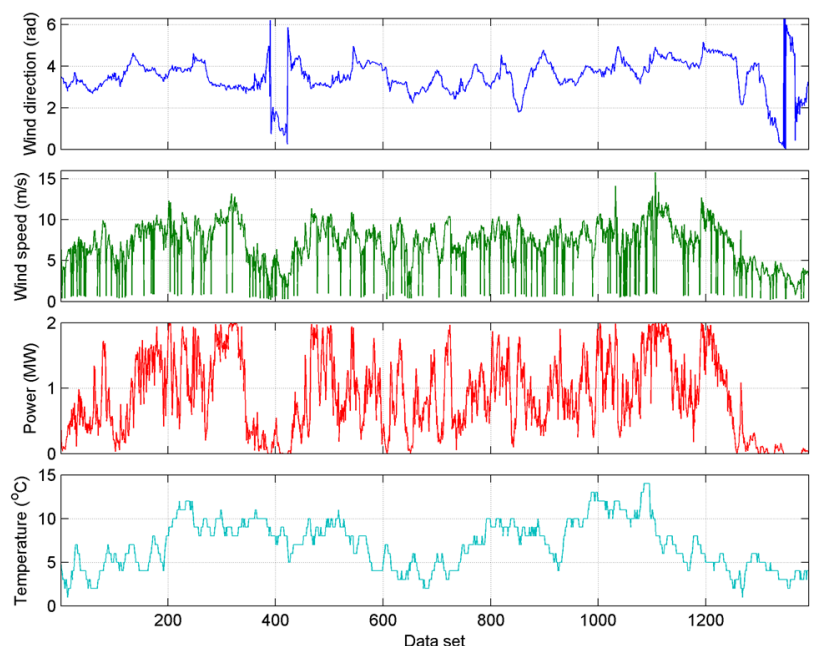

Figure 11: Long-term monitoring: Plot of the four selected input variables versus time (1392 values corresponding to the recorded vibration response datasets).

Since no damages were observed during the almost one month measurement campaign described above, a monitoring of the WT for a longer period of time, which will allow the assessment of the method and its capabilities for damage detection, is already planned. It is worth noting that SCADA data measured at higher sampling frequencies could allow the better statistical characterization of the PCE input variables potentially leading to improved performance of the method. An effort is currently underway for ensuring such a synchronized acquisition. 


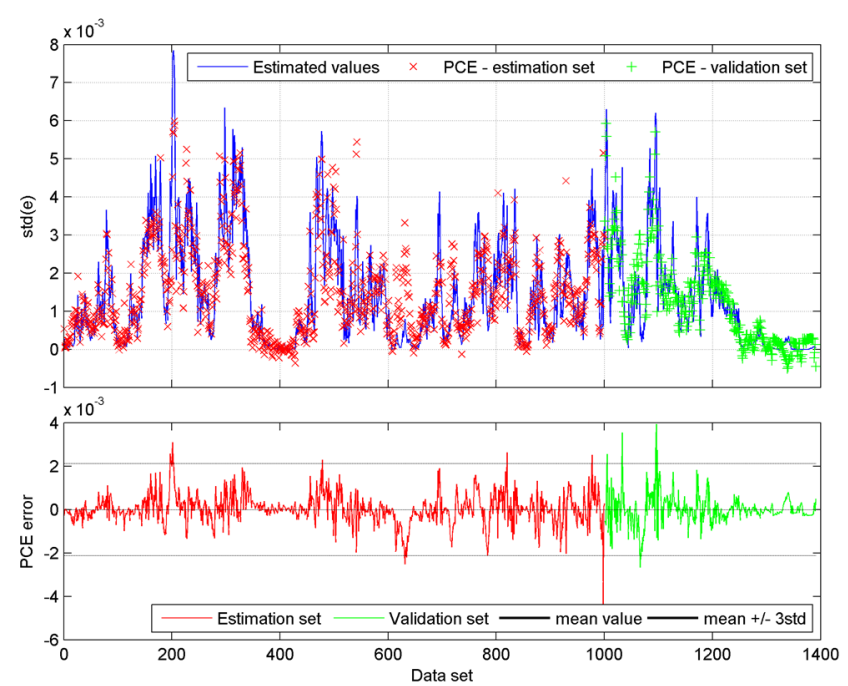

Figure 12: Long-term monitoring. SP-TARMA(32,32) model residual std as obtained from the modelling of each vibration response dataset along with the PCE model expansion values. The PCE errors along with their 95\% confidence intervals are plotted in the bottom.

\section{Conclusions}

Environmental and operational condition data, nowadays largely available in modern SHM systems of large-scale civil engineering structures, should be accounted for in favor of identifying comprehensive dynamic models. To this end, a long-term monitoring framework (PCE-ICA) is herein introduced, relying on a polynomial chaos expansion tool for the accurate description of the stochasticity emanating from external influencing agents onto structural response.

A procedure for extracting condition indicators, or performance indices, is delivered which may serve for warning of irregularity/damage on the system and which incorporates the effects of operational or environmental influences. This component requires the operation of a low-cost monitoring system over a long period of time but can provide significant information concerning the influence of external factors on structural performance, by simply relying on ambient (operational) response data. Once this influence is quantified, a simple indicator may be extracted able to indicate whether the structure's condition lies within regular bounds or whether, to the contrary, a deviation indicating damage, or deterioration is tractable. Application of the method on the two actual large-scale civil case studies demonstrates the potential of adoption of the proposed framework into practice.

\section{ACKNOWLEDGMENTS}

The authors would like to gratefully acknowledge Prof. Edwin Reynders and Prof. Guido De Roeck for providing the SIMCES project data on the Z24 bridge. We additionally would like to thank Mr. Markus Romberg from Repower Deutschland GmbH, Germany for enabling the deployment in the wind turbine facility in Lübbenau, Germany as well as the handling of the data. Finally, we would like to thank the Bundesamt für Strassen - ASTRA (Federal Roads Office - FEDRO), for Research Project \# AGB 2012/015, under the auspices of which this research was carried out.

\section{References}

1. F.P. Kopsaftopoulos, S.D. Fassois, Mech Syst Signal Process, 39, 143-161, (2013)

2. H. Sohn, Philosophical Transactions of the Royal Society A, 365, 539-560, (2007)

3. B. Peeters, J. Maeck, G. De Roeck, Smart Materials and Structures, 10, 518-527 (2001)

4. B. Peeters, G. De Roeck, Earthquake Engineering and Structural Dynamics, 30, 149-171 (2001)

5. C. Farrar, S. Doebling, P. Cornwell, E. Straser, 15th International Modal Analysis Conference (IMAC XV), Orlando, USA (1997)

6. S. Alampalli, Mechanical Systems and Signal Processing, 14, 63-74 (2000)

7. F.N. Catbas, M. Susoy, D.M. Frangopol, Engineering Structures, 30, 2347-2359 (2008)

8. E.J. Cross, K.Y. Koo, J.M.W. Brownjohn, K. Worden, Mechanical Systems and Signal Processing, 35, 16-34 (2013)

9. K.-V. Yuen, S.-C. Kuok, Engineering Structures, 32, 2379-2386 (2010)

10. J. Kullaa, in New Trends in Vibration Based Structural Health Monitoring, A. Deraemaeker and K. Worden (Eds.), CISM Courses and Lectures, 520, 107-181, Springer (2011)

11. G.H. James, T.G. Carne, J.P. Lauffer, A.R. Nard, Proceedings of the 10-th IMAC, San Diego, CA, USA (1992)

12. L. Zhang, R. Brincker, Proceedings of the 1st International Operational Modal Analysis Conference, Copenhagen, Denmark (2005)

13. E. Reynders, G. De Roeck, Encyclopedia of Earthquake Engineering, edited by M. Beer, I. A. Kougioumtzoglou, E. Patelli, S-K. Au, Springer Berlin Heidelberg, 1-9 (2014)

14. B. Peeters, G. De Roeck, ASME Journal of Dynamic Systems, Measurement, and Control, 123, 4, 659-667 (2001)

15. F. Magalhaes, Á. Cunha,Mechanical Systems and Signal Processing, 25, 5,1431-1450 (2011)

16. M. Spiridonakos, A. Poulimenos, S. Fassois, Journal of Sound and Vibration, 329, 768-785 (2010)

17. M. Spiridonakos, E. Chatzi, Proceedings of ECCOMAS Thematic Conference - COMPDYN 2013: 4th International Conference on Computational Methods in Structural Dynamics and Earthquake Engineering, 1450-1459 (2013)

18. R.G. Ghanem, P.D. Spanos, Stochastic Finite Elements: A Spectral Approach, Dover Civil and Mechanical Engineering (2012)

19. G. Blatman, B. Sudret, Probabilistic Engineering Mechanics, 25, 183-197 (2010)

20. E. Reynders, G. De Roeck, in Encyclopedia of Structural Health Monitoring, C. Boller, F.-K. Chang, and Y. Fujino (Eds.), John Wiley \& Sons (2009) 
21. A. Hyvärinen, E. Oja, Neural Networks, 13, 411-430, (2000)

22. D. Xiu, G. Karniadakis, SIAM Journal on Scientific Computing, 24, 619-644 (2002)

23. C. Krämer, C.A.M. De Smet, and G. De Roeck, $17^{\text {th }}$ International Modal Analysis Conference (IMAC XVII), Kissimmee, USA (1999)

24. M. Spiridonakos, Y. Ou, E. Chatzi, M. Romberg, Proceedings of the 7th International conference on Structural Health Monitoring of Intelligent Infrastructure (SHM-II), Torino, Italy (2015)

25. M. Spiridonakos, E. Chatzi, In: Beer M., Patelli E., Kougioumtzoglou I., Au I. (Ed.) Encyclopedia of Earthquake Engineering: SpringerReference, Springer-Verlag Berlin Heidelberg (2014)

26. M.D. Spiridonakos, V.K. Dertimanis, E.N. Chatzi, CIVIL-SOFT-COMP 2015, Proceedings of the Fourth International Conference on Soft Computing Technology in Civil, Structural and Environmental Engineering, Prague, Czech Republic (2015)

27. M. Spiridonakos, E. Chatzi, Proceedings of Eurodyn 2014, 9th International Conference on Structural Dynamics, Porto, Portugal (2014)

28. M. Spiridonakos, E.N. Chatzi, "Metamodeling of Dynamic Nonlinear Structural Systems through Polynomial Chaos NARX Models", Computers and Structures, 157, 99-113, (2015)

29. M. Spiridonakos, E.N. Chatzi, SRESA Journal of Life Cycle Reliability and Safety Engineering, accepted for publication (2015) 